AIAA 2001-2185

Computational Analysis of a Pylon-Chevron Core Nozzle Interaction

R.H.Thomas, K.W.Kinzie, and S. Paul Pao

NASA Langley Research Center

Hampton, VA USA

$7^{\text {th }}$ AIAA/CEAS Aeroacoustics Conference 28-30 May 2001 Maastricht, The Netherlands 
$=$ 


\title{
COMPUTATIONAL ANALYSIS OF A PYLON-CHEVRON CORE NOZZLE INTERACTION
}

\author{
Russell H. Thomas" \\ Aeroacoustics Branch, MS 166, R.H.Thomas@larc.nasa.gov \\ Kevin W. Kinzie \\ Aeroacoustics Branch, MS 166, K.W.Kinzie@ Iarc.nasa.gov \\ And \\ S. Paul Pao* \\ Configuration Aerodynamics Branch, MS 499, S.P.Pao@Iarc.nasa.gov \\ NASA Langley Research Center \\ Hampton, VA 23681-2199
}

\begin{abstract}
$\underline{\text { Abstract }}$
In typical engine installations, the pylon of an engine creates a flow disturbance that interacts with the engine exhaust flow. This interaction of the pylon with the exhaust flow from a dual stream nozzle was studied computationally. The dual stream nozzle simulates an engine with a bypass ratio of five. A total of five configurations were simulated all at the take-off operating point. All computations were performed using the structured PAB3D code which solves the steady, compressible, Reynolds-averaged NavierStokes equations. These configurations included a core nozzle with eight chevron noise reduction devices built into the nozzle trailing edge. Baseline cases had no chevron devices and were run with a pylon and without a pylon. Cases with the chevron were also studied with and without the pylon. Another case was run with the chevron rotated relative to the pylon. The fan nozzle did not have chevron devices attached. Solutions showed that the effect of the pylon is to distort the round jet plume and to destroy the symmetrical lobed pattern created by the core chevrons. Several overall flow field quantities were calculated that might be used in extensions of this work to find flow field parameters that correlate with changes in noise.
\end{abstract}

Introduction

The integration of propulsion and airframe is a vital consideration in the design of an aircraft system. Many considerations influence the integration, such as structural, aerodynamic, and maintenance factors. Typically for major commercial airliners, engine installation involves the engine-under-the-wing or the tail mounted configuration. However, even within those two basic categories there are many design variables. For these standard engine installations, industry has highly developed design and analysis processes. However, these processes are normally finely tuned to conventional, traditional installations. Non-conventional installations pose a challenge to most of these tools. Early ${ }^{j}$ also describes some of the future challenges facing industry in the area of propulsion airframe integration design and analysis.

The impact of the propulsion airframe integration on the net radiated noise of the aircraft is one of these challenges that will be emerging as increasingly important and is the motivation for the work reported here. Whether an engine is mounted above a wing or below can have a significant effect on noise that reaches communities below. This is an

\footnotetext{
* Aerospace Engineer, Senior Member AIAA

* Senior Research Scientist, Associate Fellow AIAA

Copyright $(2001$ by the American Institute of Aeronautics and Astronautics, Inc. No copyright is asserted in the United States under Title 17, U.S. Code. The U.S. Government has a royalty-free license to exercise all rights under the copyright claimed herein for governmental purposes. All other rights are reserved by the copyright owner.
} 
obvious example of the acoustic effects of propulsion airframe installation. Another example could be the effect of the pylon on the development of the exhaust plume and on the resulting jet noise.

Another area of installation effect on noise is the impact that installation has on noise reduction devices. Many noise reduction devices are studied fundamentally on isolated components. In general, installation effects must be considered because these effects can alter the aerodynamic and noise reduction performance of the device or strategy. This focus on both the aerodynamic as well as acoustic interaction effects of installation, propulsion airframe aeroacoustics, will become more important as noise reduction targets become more difficult to achieve. These more stringent noise reduction targets will require, in addition to continued fundamental component reduction efforts, a system level approach that includes propulsion airframe acroacoustics.

The work in this paper is the beginning of a larger effort to study one specific effect within this broad category, that of the pylon on separate flow nozzles including those equipped with the chevron jet noise reduction device. The results presented here are preliminary computational results that will be used in future research together with more computational studies, experiments and jet noise prediction methods.

Chevron nozzles have been extensively studied in recent years and have proven to be a promising jet noise reduction method because the accompanying thrust loss is low enough to be economically viable. Chevrons work by altering the mixing characteristics of a jet resulting in reduction of the overall noise levels radiated from the jet. More work is required to develop an exact understanding of the chevron noise reduction mechanism.

During the NASA Advanced Subsonic Transport (AST) program, chevrons were tested extensively at the Glenn Research Center with industry participation. The separate flow nozzles were tested as isolated components, without an attached pylon. The best chevron designs from this test matrix showed a 2.7 EPNdB noise reduction at take-off conditions with a 0.06 of a percentage point drop in thrust coefficient at cruise $^{2}$. These figures are representative of chevron noise reduction potential with the current level of understanding.

In parallel with the Glenn studies was a computational study by Kenzakowski et al. ${ }^{3}$ on many of the same chevron designs and on tab mixing devices. Good agreement between calculations and experiments was shown. In order to improve the comparison, Kenzakowski et al. recommended including upstream nozzle effects, a variable Prandtl number, and using non-linear extensions to the baseline $k-\varepsilon$ turbulence model to model anisotropic effects as suggested by
Khavaran ${ }^{4}$. These suggestions could be important for finer comparison of experiment and computation.

Calculations such as those by Kenzakowski and those in this paper represent significant opportunity for investigation of the flow field characteristics and also to serve as a beginning point of a more comprehensive investigation. Therefore, one of the primary objectives of this work is to begin to develop an understanding of the characteristics of the flow field of the selected installation configurations. In future steps, these calculations will be used in jet noise prediction methods that are based on computational solutions. Experiments will also be performed at the Jet Noise Laboratory of NASA Langley on these same configurations. Together, these three tools will be used to develop an understanding of the acoustic interaction between pylon and chevron devices and the more general interaction of pylon and jet. Therefore, the work presented here represents an initial study into installation effects involving the pylon.

\section{Configuration List}

The baseline configuration is a separate flow nozzle at a bypass ratio of five with an external plug. This nozzle was tested at NASA Langley during the AST program in parallel with the NASA Glenn tests ${ }^{2}$. The pylon was taken from a nozzle design study performed at McDonnell Douglas in 1996 and represents a generic pylon. The chevrons were designed for the core nozzle using guidelines similar to those used in the AST program. Chevron numbers from four to twelve were considered with only results for eight chevrons being presented in this work. The chevrons were designed to penetrate into the core flow by approximately the thickness of the boundary layer. The trailing edge of the baseline nozzle was chosen to correspond to the mid-point of the chevron axial length. The five configurations analyzed in this study are:

1. Baseline round core and fan nozzle with no pylon (figure 1).

2. Baseline core and fan nozzle with pylon.

3. Chevron core nozzle and baseline fan nozzle with no pylon.

4. Chevron core nozzle and baseline fan nozzle with pylon (figure 2 ).

5. Configuration same as number 4 but with the core chevron clocked $1 / 2$ of the wavelength of a chevron.

Figure 3 shows the junction of the pylon and the core nozzle with chevrons of configuration 4 . Also shown are the surface pressure contours calculated from the procedure to be described in the following section. This junction is simply the result of a separate chevron and pylon design combined with no added design 
feature for the junction. This can be compared with the junction of configuration 5 that is shown in figure 4 .

The purpose of configuration 5 is to produce a different arrangement between the core chevron and the pylon. In configuration 4 a tip of a chevron is directly beneath the centerline of the pylon but in configuration 5 a trough of a chevron is underneath the pylon. This is accomplished by rotating the chevron trailing edge by one-half the wavelength of a chevron. Figures 3 and 4 show through the surface streamlines the immediate effect of the two chevron-pylon orientations on the flow in the immediate vicinity of the pylon junction. Configuration 4 (figure 3) produces a separation bubble on the underside of the pylon, the pylon shelf. This is because the chevron design includes penetration into the core flow that creates a cavity. This cavity is still present in configuration 5 however it is reduced with the disturbance to the flow on the pylon also being reduced. The orientation of the chevrons is identical between configurations 3 and 4 .

\section{$\underline{\text { Simulation Conditions }}$}

The computations for these five configurations were at the take-off condition on a generic cycle line from the same McDonnell Douglas AST study. The freestream total conditions were set at a pressure of the standard sea level atmosphere and a temperature of 295 $\mathrm{K}$ with a Mach number 0.28 . The fan was set at a nozzle pressure ratio of 1.75 and a total temperature of $350 \mathrm{~K}$. The core nozzle pressure ratio was 1.56 with a total temperature of $828 \mathrm{~K}$.

\section{Computational Approach}

The computational domain for the solution extended from $x / D_{c}=-6.3$ to $x / D_{c}=31.6$ in the axial direction and $6.3 \mathrm{D}_{\mathrm{c}}$ in the radial direction, where $\mathrm{D}_{c}$ is the diameter of the baseline core nozzle, $12.80 \mathrm{~cm}$. The origin, $x / D_{c}=0.0$ was set at the exit of the fan nozzle so that the exit of the core nozzle is at about $\mathrm{x} / \mathrm{D}_{\mathrm{c}}=0.5$. Since the solution procedure called for two solution types, the grid size is given for both regions in Table 1 as well as a total grid size for each case. Because of the three dimensional nature of the problem, the grids covered 180 degrees in the azimuthal direction of the nozzle as shown in figures 1 and 2 .

Figure 5 shows the layout of the grid boundaries in relation to the nozzle and the approximate plume boundary. The plume boundary is identified at the point where, scanning radially at a given axial station, the total temperature drops to within 0.5 percent of the freestream value of $295 \mathrm{~K}$. Figure 5 shows the plume boundary for the baseline case, configuration 1 . Also shown are the planes where cross sections of flow quantities were examined, those at $x / D_{c}=2,5$, and 8 that will be shown in the following sections.

Table 1. Grid Size in Terms of Number of Cells.

\begin{tabular}{|c|r|r|r|}
\hline $\begin{array}{l}\text { Configuration } \\
\text { Number }\end{array}$ & $\begin{array}{l}\text { Time } \\
\text { Marched } \\
\text { Grid }\end{array}$ & $\begin{array}{l}\text { Space } \\
\text { Marched } \\
\text { Grid }\end{array}$ & $\begin{array}{l}\text { Total Cell } \\
\text { Count }\end{array}$ \\
\hline 1 & $4,083,072$ & $1,797,120$ & $5,880,192$ \\
\hline 2 & $7,805,440$ & $3,594,240$ & $11,399,680$ \\
\hline 3 & $10,125,312$ & $3,594,240$ & $13,719,552$ \\
\hline 4 & $12,620,096$ & $3,594,240$ & $16,214,336$ \\
\hline 5 & $13,383,744$ & $3,594,240$ & $16,977,984$ \\
\hline
\end{tabular}

The flow was simulated with the asymptotically steady, compressible, Reynoldsaveraged Navier-Stokes equations using an implicit, upwind, flux-difference splitting finite volume scheme. A standard two-equation $k-\varepsilon$ turbulence model with a linear stress representation was used. The solution procedure was implemented with the multi-block, parallel, and structured code PAB3D ${ }^{5}$. Additional methods used were the flux difference splitting scheme of Roe, uncoupled viscosity in the normal and circumferential directions, and a two-factor scheme for the approximation of implicit terms.

The general solution strategy was to solve the region near the nozzle through time marching followed by solving the downstream jet plume with space marching. With solutions in both regions, a final procedure was to run additional iterations over both regions with time marching to smooth any inflections at the boundary between the time marching and space marching regions. The solutions were obtained on PC parallel clusters of between 4 and 8 machines and were done in three steps. Initial run times ranged from 50 to 150 hours for time marching near the nozzle, depending on the configuration, followed hy 3-6 hours for space marching the downstream plume. A final procedure to time march the whole plume took 4-8 hours.

\section{$\underline{\text { Simulation Results }}$}

The first results presented are the total temperature contour plots in the centerline plane for all five configurations (figure 6a-e). In this figure the freestream total temperature of $295 \mathrm{~K}$ is set to the color black in order to highlight the jet plume. The total temperature contours in the vertical symmetry planc shown in figure 6 are useful for identifying the plume boundary and also the potential core of the core jet that is identified by the red color corresponding to core temperatures above $800 \mathrm{~K}$. Figure 6 is arranged such that parts a through e correspond to configurations 1 through 5 . In this arrangement, configuration I for the 
baseline nozzle without a pylon (figure $6 \mathrm{a}$ ) has the longest potential core extending out to about $13 \mathrm{D}_{\mathrm{c}}$. The addition of the pylon, configuration 2 in figure $6 \mathrm{~b}$, appears to shorten the potential core to about $1 / D_{c}$. The chevron core nozzle has a dramatic effect in figure $6 c$ where the potential core is reduced to $10 \mathrm{D}_{\mathrm{c}}$ or less and the core appears much smaller in radius compared to the baseline case. The addition of the pylon to the chevron nozzle in configurations 4 and 5 accentuates the three dimensionality of the flow field. For these cases an accurate measure of the potential core length comes from the plot of maximum temperature that will be shown in a figure near the end of this paper.

Both the pylon and the chevrons introduce a three dimensional structure to the jet and it is therefore important to remember that one particular section cut will not reveal the full three dimensionality of the jet. That is the case with the vertical centerline plots as will be illustrated by comparing these plots with the cross sections to be presented in the following figures.

In figure 7 cross sections of total temperature for three downstream stations, $x / D_{c}=2,5$, and 8 , are shown for all five configurations in the same order, from $7 \mathrm{a}$ to $7 \mathrm{c}$, as in figure 6 .

The chevron nozzle introduces a pairing of vortices off adjacent chevrons that produce the lobes that are clearly seen in figure $7 \mathrm{c}$ for the configuration with the chevron core nozzle without pylon. Compared to figure $7 \mathrm{a}$ for the baseline nozzle, it is clearly seen again how the chevron nozzle increases the mixing rate over the baseline nozzle with a much smaller core jet left by $x / D_{c}=8$.

The pylon also introduces a three dimensional aspect to the flow field as seen in figure $7 \mathrm{~b}$ for the round nozzle with pylon. The effect of the pylon, comparing $7 \mathrm{a}$ to $7 \mathrm{~b}$, is to stretch the jet in the vertical direction making the shape of the core jet more triangular than circular and the shape of the fan jet more elliptical than circular by $x / D_{c}=8$. It is important to note that the effect of a lower bifurcation is also contributing to the distortion of the flow field. The lower bifurcation is only about $27.8 \%$ of the thickness and $25.8 \%$ of the length of the pylon. However, even with these dimensions its effects are still evident. Also, while the pylon was treated with viscous boundary conditions the lower bifurcation was treated with a nonviscous boundary condition both because its effect was expected to be much less and as a measure to reduce overall grid size. Therefore, if viscous boundary conditions were used on the lower bifurcation, its effect would be more noticeable than shown here.

The effect of the pylon on the chevron nozzle flow field for configuration 4 with a chevron tip directly under the pylon is seen in figure $7 \mathrm{~d}$. The pylon, which tends to draw the core jet outward, counteracts the effect of the chevron which, with the tip directly under the pylon, is drawing a fan lobe down into the core flow. As a result, two of the core lobes merge without fan flow separating them.

This effect is mitigated by changing the orientation of the chevron relative to the pylon, configuration 5, with a trough under the pylon instead of a peak, as seen in figure $7 \mathrm{e}$. In this orientation, seven core lobes are able to form with one core lobe directly affected by the pylon.

However, it is important to note that in both orientations of chevrons relative to the pylon that the entire chevron pattern is affected by the distortion introduced by the pylon. This is clearly seen by comparing figures $7 \mathrm{~d}$ and $7 \mathrm{e}$ to $7 \mathrm{c}$. In both figures $7 \mathrm{~d}$ and $7 \mathrm{e}$, downstream at $\mathrm{x} / \mathrm{D}_{\mathrm{c}}=5$ and 8 , the pylon causes the core lobes to migrate in an asymmetrical manner as opposed to the symmetry of the core lobes in figure $7 \mathrm{c}$.

We now turn our attention to the effects shown by the five configurations on the distribution and levels of turbulence kinetic energy (TKE). The cross sections of TKE are shown in figure 8a-e which is in the same format as figure 7 . Figure 8 a shows the TKE for the baseline case at the same three downstream stations. The outer shear layer due to the fan-freestream mixing layer is shown to be not only thicker radially by $x / D_{c}=$ 2 but also has noticeably higher levels of TKE compared to the shear layer created by fan-core mixing. On the axis the wake created by the plug can be seen.

The effects of the pylon are seen in figure $8 b$. The fan-core mixing layer is more noticeably distorted into the triangular shape that was also clear in figure $7 \mathrm{~b}$. The fan-freestream layer is also distorted but to a lesser extent.

The most significant effect in figure $8 \mathrm{~b}$ is the localized region of high TKE that is clear at $x / D_{c}=5$ and 8 . It appears to form at the location where the corefan mixing layer on both sides of the pylon merges downstream of the trailing edge of the pylon. The combination of chevrons with the pylon, figure $8 \mathrm{~d}$, show that same high TKE region at the same location, although it has spread in the radial direction. However, when the chevrons are rotated the high TKE region is removed completely, figure $8 \mathrm{e}$.

Together with the experiments and noise predictions, when they are available, differences between the five configurations like the high TKE regions discussed above can begin to provide an understanding of the characteristics of the nozzle configurations and how they might relate to noise generation. In addition, extracting mass averaged quantities from the flow field solutions is another way of seeking quantities that can discriminate between the various configurations in a way that may eventually relate to noise characteristics.

Mass averaged total temperature as a function of axial distance is shown in figure 9 for all five 
configurations. The mass averaged temperature is computed by integrating the product of total temperature and mass flow and then dividing by the integral of the mass flow, both over the same area. The area of integration at an axial station is in the radial direction to the point where temperature is within 0.5 percent of the freestream value. There is very little difference between the five configurations with only about $5 \mathrm{~K}$ difference between the configurations at axial stations up to $\mathrm{x} / \mathrm{D}_{\mathrm{c}}=7$. This is somewhat surprising considering the dramatic difference in the total temperature contours shown between the configurations in figure 7. However, the chosen integration limit results in mass averaging over the entire jet which is heavily weighted by the fan stream due to the high bypass ratio. If the limit of integration in the radial direction is set at 0.5-percent of the fan stream temperature then the mass averaging will take place over primarily the core jet. This is the limit used in figure 10 . It should be noted that with a limit defined by temperature the mass averaging will take place over an area that follows the shape of the core jet even when it has a lobed pattern such as in figure $7 \mathrm{c}$. Also, in the axial direction, the mass averaging will not be possible when the core jet temperature falls below 1.005 of the fan temperature which occurs at about $x / D_{c}=27$ in figure 10. Configurations 3-5 that have chevrons show a much faster drop in mass averaged total temperature in figure 10 as compared to configurations 1-2 that are the round nozzles. The results in figure 10 compare qualitatively very well with figure 22 of Kenzakowski et al. ${ }^{3}$ using a similar mass averaging parameter that was limited to the core jet. Therefore, it appears that the chevrons primarily affect the mixing between the fan and core jets and have little impact on the mixing of the fan with freestream.

The overall entrainment of the jet can also be assessed by calculating the total mass flow in the jet at a given axial station normalized by the mass flow at the exit of the nozzle. This normalized jet mass flow is shown in figure 11 and confirms that the overall mixing for the entire jet is similar for all five configurations and is relatively unaffected by either the pylon or the chevron mixing enhancement devices. Both of the quantities shown in figures 9 and 11 reflect overall mixing of the plume with the freestream that is primarily determined by the mixing between the fan stream and the freestream.

Mass averaged TKE (see figure 12) is calculated over the whole jet cross sectional area, out to the point where total temperature is at 0.5 -percent of the freestream total temperature. Figure 12 shows that this quantity can vary significantly for the five configurations. Configurations $I$ and 2, nozzles without chevrons, show the highest peak levels of mass averaged TKE between $x / D_{c}=10$ and 15 .
Configuration 3 that adds the chevron core nozzle shows a broad peak extending from $x / D_{c}=5$ to 15 . Adding the pylon to the chevron nozzle, configurations 4 and 5, lowers the level of mass averaged TKE over the whole jet plume. An interesting feature of all three configurations with chevrons is that there is an abrupt change in the mass averaged TKE at about $x / D_{c}=6$.

Mass averaged vorticity is shown in figure 13 and is another quantity that shows some key differences between the configurations. Initially, all configurations show high levels of vorticity within the first two diameters. The differences are clearly seen in the region from the nozzle exil and up to $x / \mathrm{D}_{c}=6$. As expected the round nozzle has the lowest level of vorticity. The addition of the pylon, configuration 2 , increases the vorticity level, however, all three configurations with chevrons have significantly higher Ievels over either configuration 1 or 2 . In the region beyond $\mathrm{x} / \mathrm{D}_{\mathrm{c}}=6$ the vorticity levels and decay rates all return to similar trends.

For this limited set of parameters studied it appears that mass averaged quantities can be used to quantify differences between the configurations. Limiting the mass averaging to the core jet is one way of identifying the effect of configurations that interact primarily with the core-fan mixing layer. Parameters that are related to the entrainment of the freestream by the overall jet plume will be relatively unaffected by the effects of core chevron and pylon configurations. However, some parameters that are more directly related to characterizing the turbulent mixing process, TKE and vorticity for example, show configuration dependent characteristics even when mass averaged over the whole plume.

A final tool examined in this study was to plot the maximum levels of various quantities at given axial stations even though the radial location can vary from station to station. For total temperature this results in a classic decay plot shown in figure 14. Since it is the maximum total temperature at a station, this plot primarily reflects the mixing of the core stream. The five cases have clearly different decay rates out to about $\mathrm{x} / \mathrm{D}_{\mathrm{c}}=25$. Also, the length of the potential core is identified off of this decay plot and is shown in Table 2.

Table 2. Length of Potential Core in Core Jet Diameters for All Five Configurations, Taken from Figure 13.

\begin{tabular}{|l|c|}
\hline Configuration Number & $\begin{array}{l}\text { Length of Potential Core } \\
\text { in Core Jet Diameters, } D_{c}\end{array}$ \\
\hline 1, round & 13 \\
\hline 2, round with pylon & 11 \\
\hline 3, chevron & 8 \\
\hline 4, chevron with pylon & 5 \\
\hline $\begin{array}{l}5, \text { clocked chevron with } \\
\text { pylon }\end{array}$ & 6 \\
\hline
\end{tabular}


Figure 15 displays how the maximum TKE level at given axial stations are distributed for the five configurations. For the round nozzle, the addition of a pylon increases the maximum level of TKE and moves the location of the maximum upstream. For the chevron nozzle the addition of the pylon (configuration 4) has the effect of raising the level of maximum TKE. The clocked chevron configuration 5 raises the level of TKE much less, so the clocking of the chevron relative to the pylon changes the level and the location of the maximum TKE.

\section{Discussion of Results and Status}

Five configurations of separate flow nozzles were computationally investigated with RANS CFD. This investigation represents the beginning of a more comprehensive study of installation effects on jet noise. This computational investigation has provided a good documentation of the mean flow field of the configurations. The details of the interactions between jet and pylon are clearly seen in various flow quantities such as total temperature and turbulent kinetic energy. The effect of the pylon is to distort both the core and the fan jet with the largest effect being on the core jet. The pylon also affects the chevron jet flow field by distorting the chevron lobes around the whole circumference. The orientation of the chevrons relative to the pylon was found to have a significant effect on the development of the lobes in closest proximity to the pylon.

A goal of this research in the long term is to develop a better understanding of the connection between quantities that can be extracted from RANS CFD and the acoustic performance of the configurations. Some of these measures were reported in this paper. Measures of overall mixing, dominated by the fan-freestream mixing, showed little difference between the different configurations. However, the different configurations do have a significant impact on the momentum exchange between the fan and the core streams as evidenced by the cross section plots. The decay of maximum total temperature, which reflects the mixing of the core stream, for example, showed significant differences between configurations. Other parameters that showed significant differences between the cases were mass averaged and maximum levels of TKE and mass averaged vorticity.

Results from experiments yet to be performed will provide the information on the acoustic performance. The experiments will also provide flow freld information that can be compared to the computational results. Together with the computational results and the experiments, a jet noise prediction method based on the computational information is also being pursued. These three methods will be used concurrently in the ongoing investigation of installation effects on jet noise.

\section{Acknowledgements}

Steven Massey and Kenrick Waithe of Analytical Services and Materials are acknowledged for their roles in performing the calculations and detailed graphics. We also thank Mike Wiese of the NASA-Langley/CSC Geolab for grid construction.

\section{$\underline{\text { References }}$}

1. Early, K., "Propulsion Airframe Integration Design, Analysis and Challenges Going Into the $21^{\text {st }}$ Century," Paper No. 6103 presented at the International Council of the Aeronautical Sciences.

2. Saiyed, N.H., Mikkelsen, K.L., and Bridges, J.E., "Acoustics and Thrust of Separate-Flow Exhaust Nozzles with Mixing Devices for High-Bypass-Ratio Engines," AIAA Paper No. 2000-1961 presented at the $6^{\text {th }}$ AIAAVCEAS Aeroacoustics Conference, June, 2000.

3. Kenzakowski, D.C., Shipman, J., Dash, S.M., Bridges, J.E., and Saiyed, N.H., "Turbulence Model Study of Laboratory Jets with Mixing Enhancements for Noise Reduction," AIAA Paper 2000-0219 presented at the $38^{\text {th }}$ Aerospace Sciences Meeting, January, 2000.

4. Khavaran, A., "Role of Anisotropy in Turbulent Mixing Noise," AIAA Journal, Vol. 37, No. 7, 1999, pp. 832-41.

5. Abdol-Hamid, K., "Development of a ThreeDimensional Code for the Analysis of Jet Mixing Problems," NASA CR 4200, 1988. 


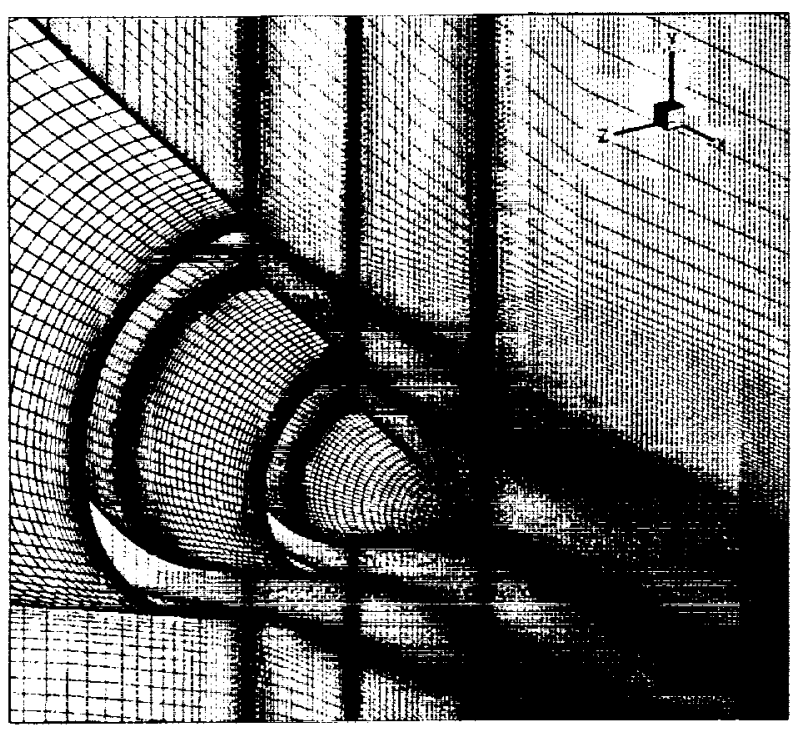

Figure 1. Computational grid of round, separate flow nozzle (configuration 1).

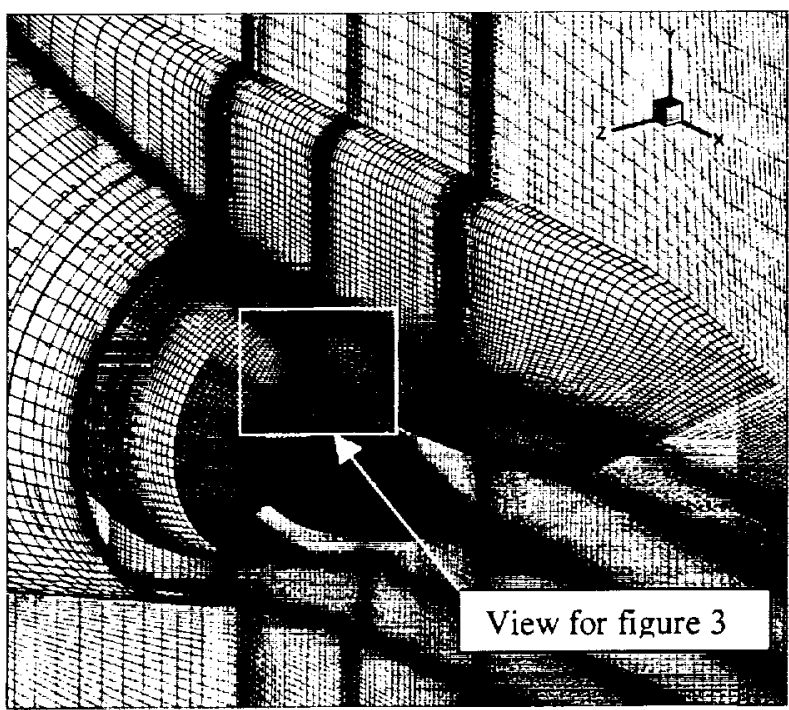

Figure 2. Computational grid of separate flow nozzle with pylon and chevron core nozzle with eight chevrons (configuration 4).

Figure 5 (to the right). Schematic of the computational boundary surrounding the nozzle with the approximate boundary of the jet identified and vertical lines identifying stations where cross sections of the flow field will be presented.

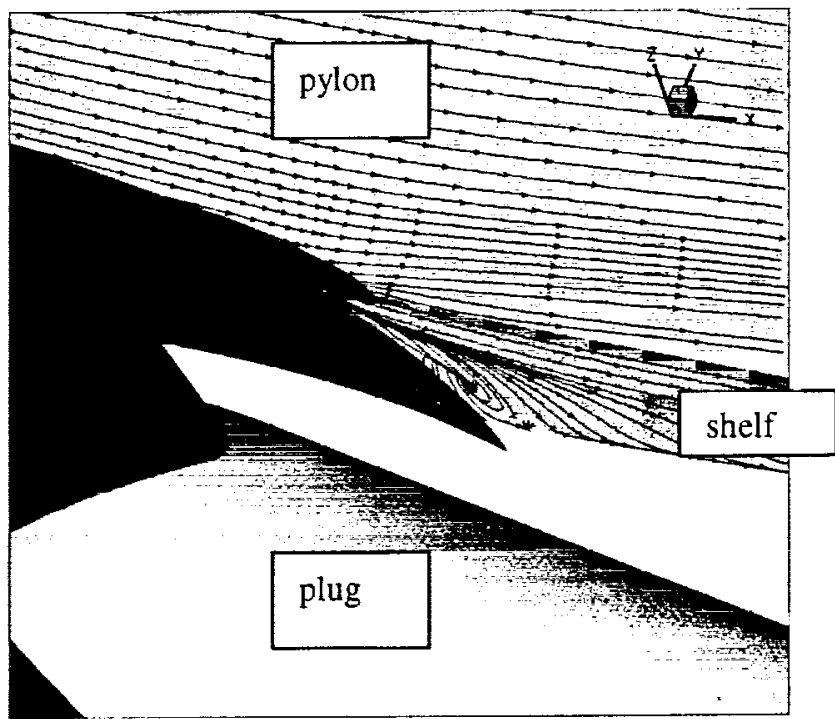

Figure 3. Surface streamlines on the pylon surfaces for the junction of the pylon and core nozzle with chevrons (configuration 4).

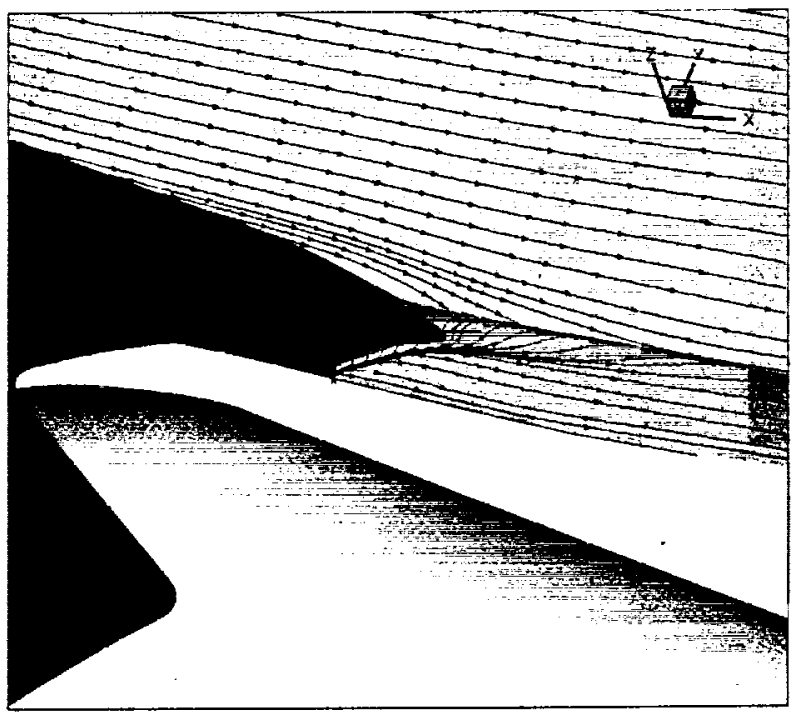

Figure 4. Surface streamlines on the pylon surfaces for the junction of the pylon and core nozzle with chevrons (configuration 5 ).

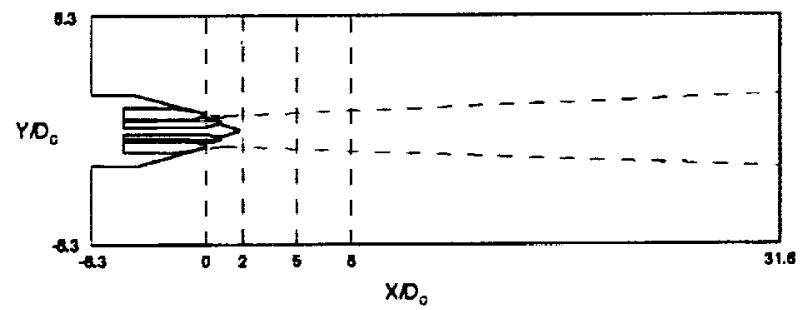


AIAA 2001-2185

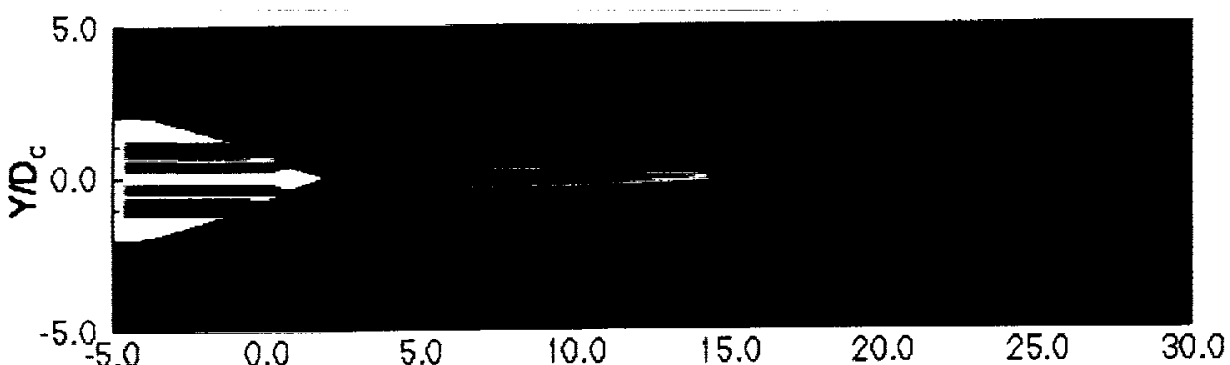

560

495

430

365

300

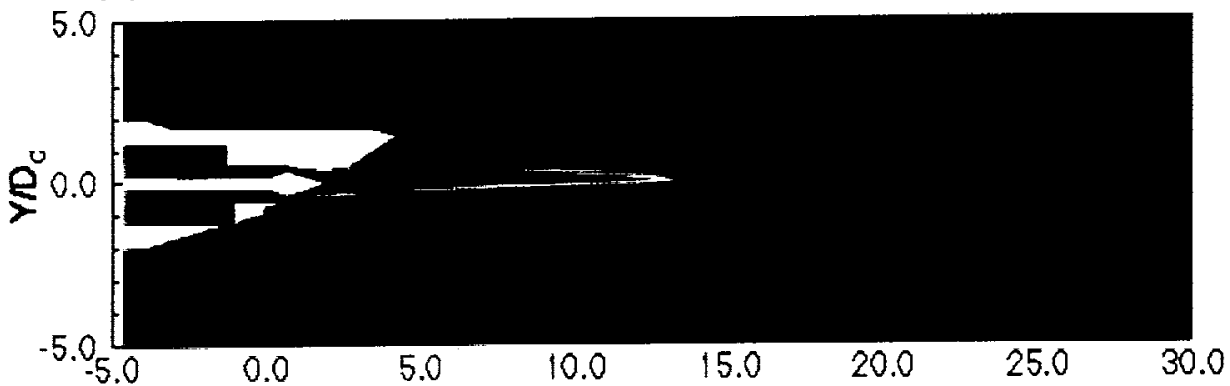

819

754

689

624

560

495

430

365

300

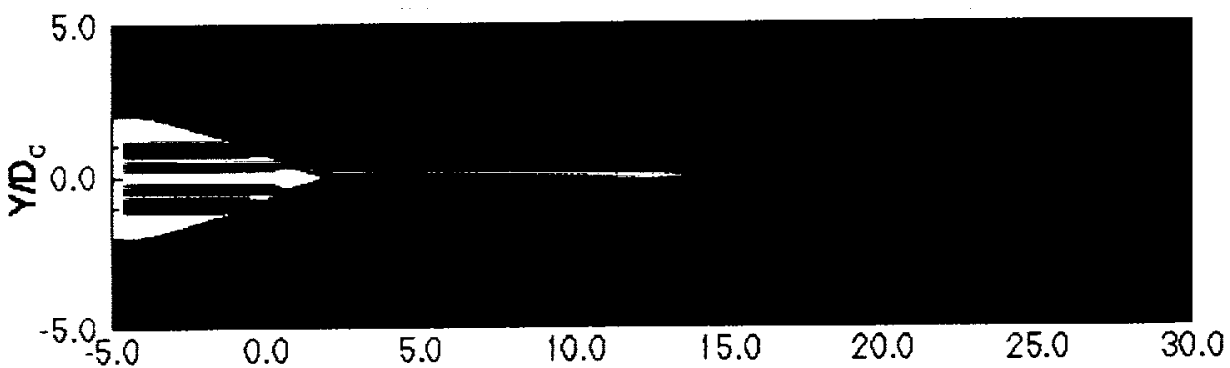

819

754

689

624

560

495

430

365

300
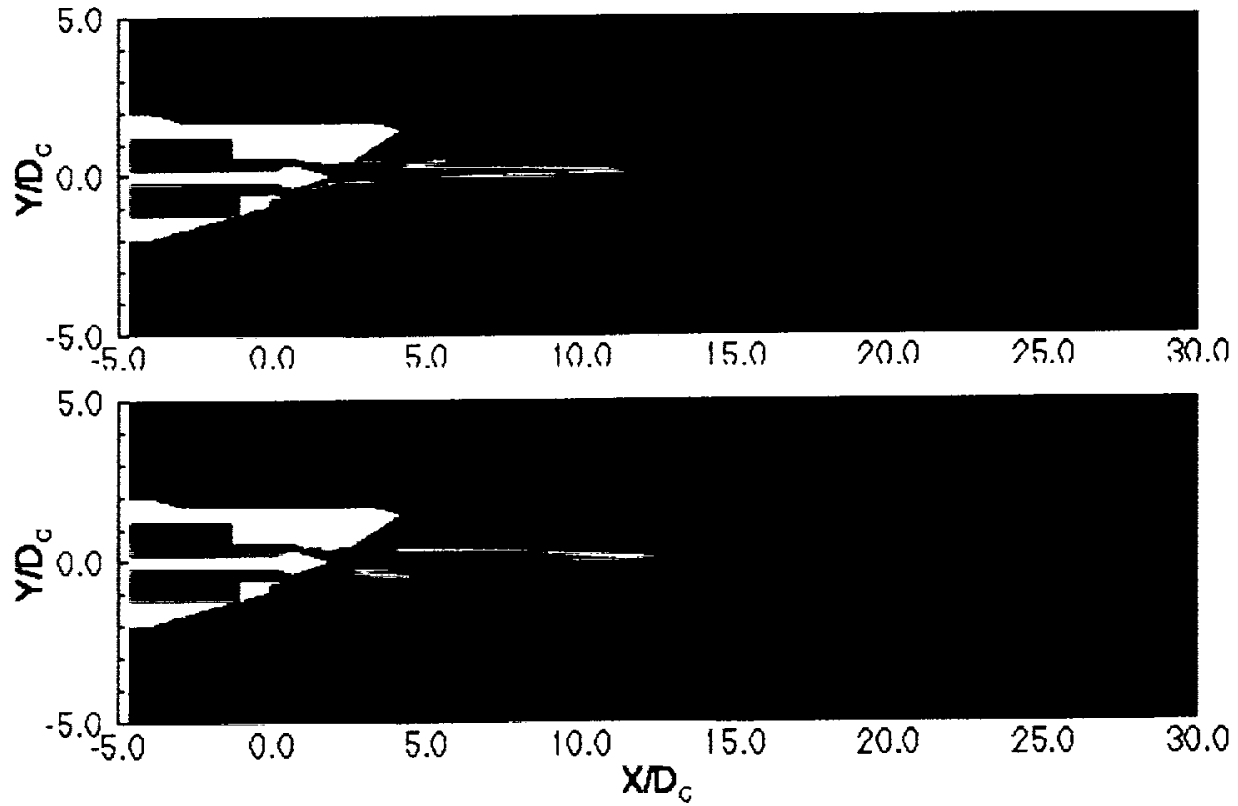

a.

Temperature in $\mathrm{K}$

c.

d.

e.

Figure 6. Centerline symmetry plane plot of total temperature $(K)$ a. round nozzle configuration \#1, b. round nozzle with pylon configuration $\# 2$, c. chevron core nozzle configuration \#3, d. chevron core nozzle with pylon configuration $\# 4$, e. chevron core nozzle clocked $1 / 2$ wavelength with pylon configuration \#5. 
$X=2 D_{c}$
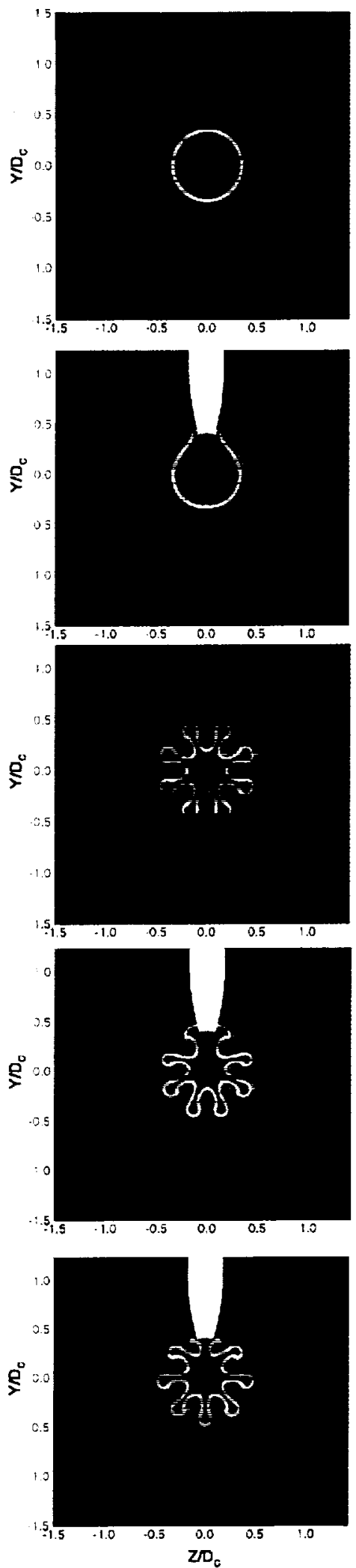

$X=5 \mathrm{D}$
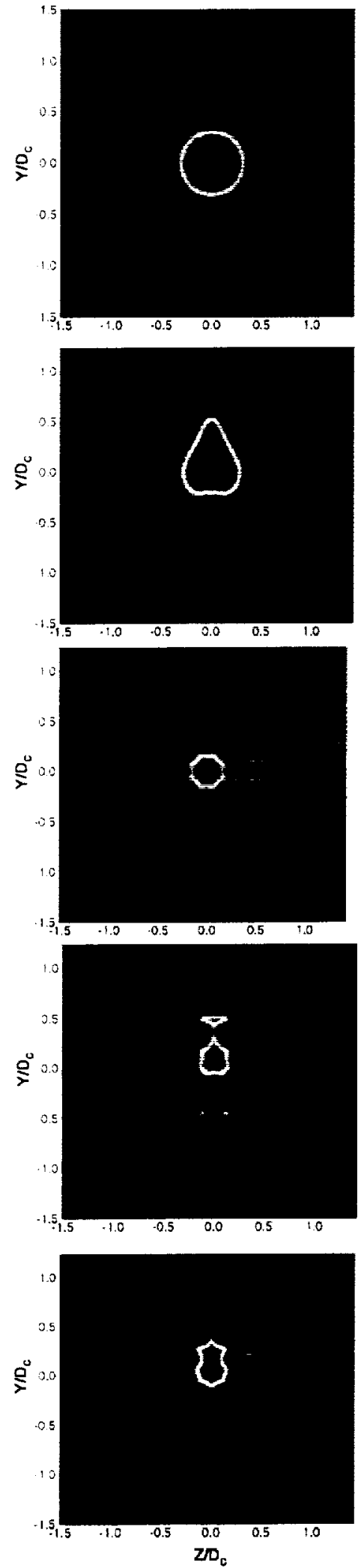

$X=B D_{c}$

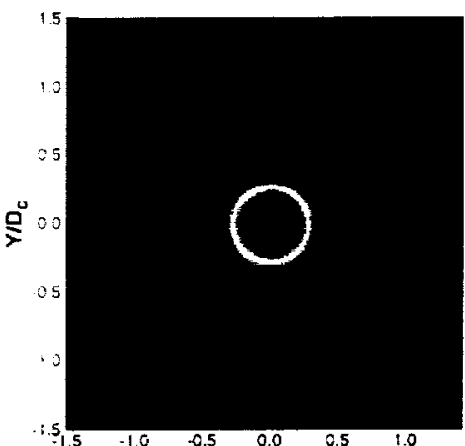

a.

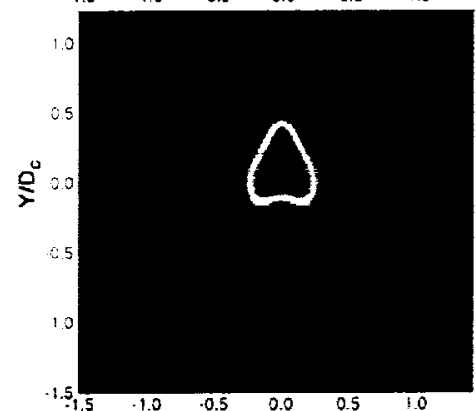

b.
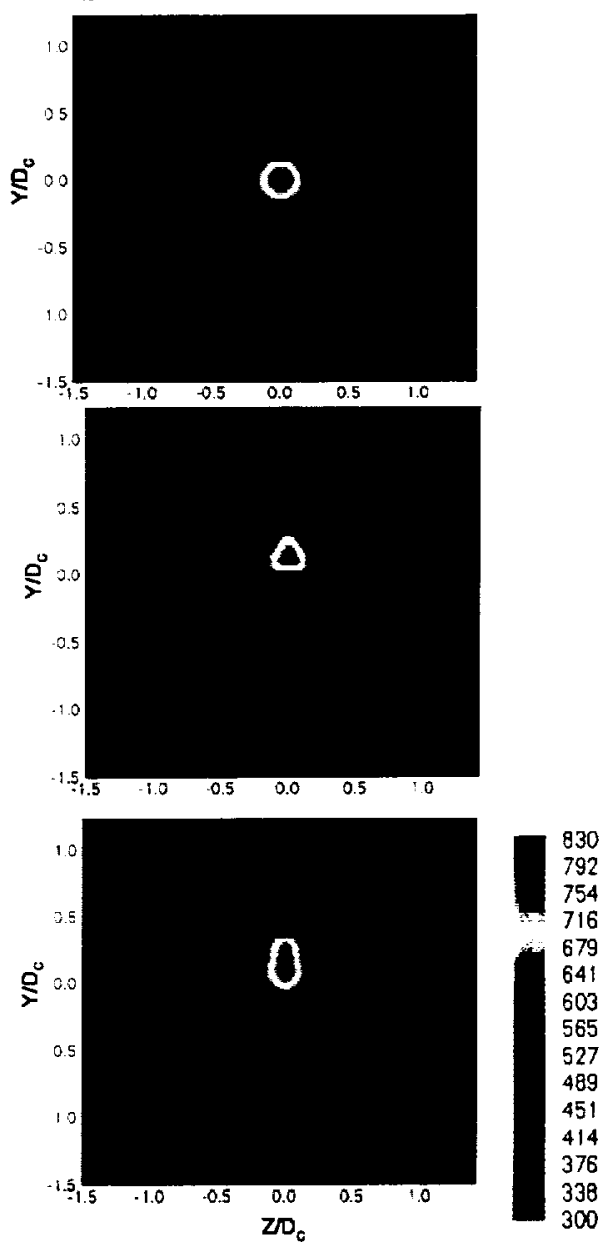

d.

c.

e.

Temperature in $\mathrm{K}$

Figure 7. Total temperature cross sections at $\mathrm{x} / \mathrm{D}$ of 2,5 , and 8 for five configurations: a. baseline $1, b$. baseline with pylon 2 , c. chevron 3 , d. chevron with pylon 4 , and e. chevron clocked $1 / 2$ wavelength with pylon. Temperature scale in degrees $K$. 
$X=2 D_{C}$
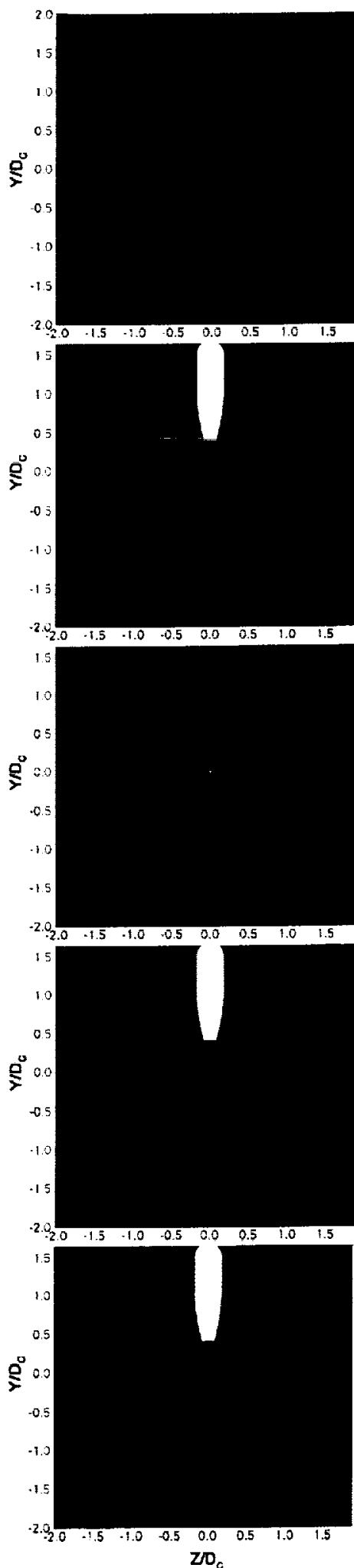

$X=5 D_{c}$
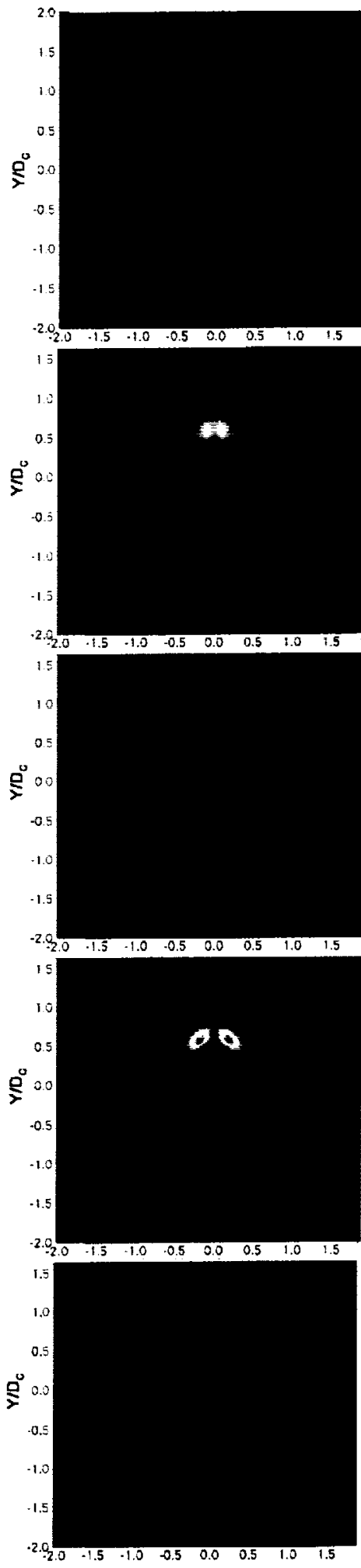

$\mathbf{Z D}_{\mathrm{c}}$
$X=8 D_{c}$

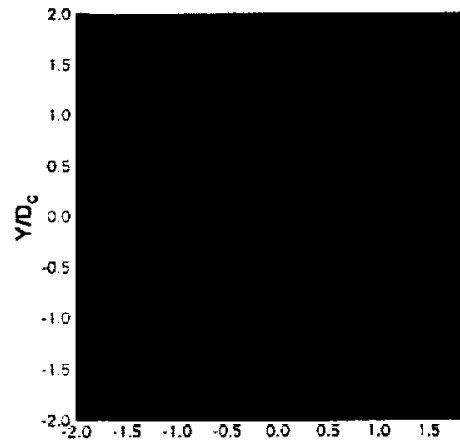

a.

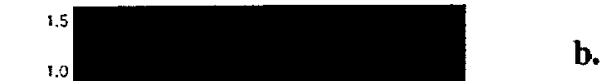

c.

d.

Figure 8. Turbulent kinetic energy cross sections at $\mathbf{X} / \mathrm{D}$ of 2,5 , and 8 for five configurations: a. baseline configuration 1 , b. baseline with pylon \#2, c. chevron \#3, d. chevron with pylon \#4, and e. chevron clocked $1 / 2$ wavelength with pylon \#5. TKE color scale in $\mathrm{KJ} / \mathrm{kg}$. 


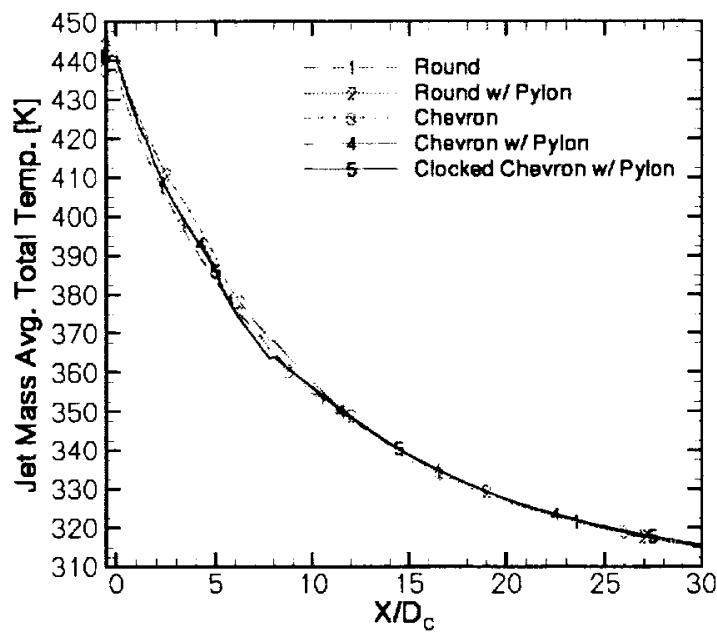

Figure 9. Mass averaged total temperature as a function of axial distance for configurations 1-5. Radial limit of integration at 0.5 -percent of ambient temperature.

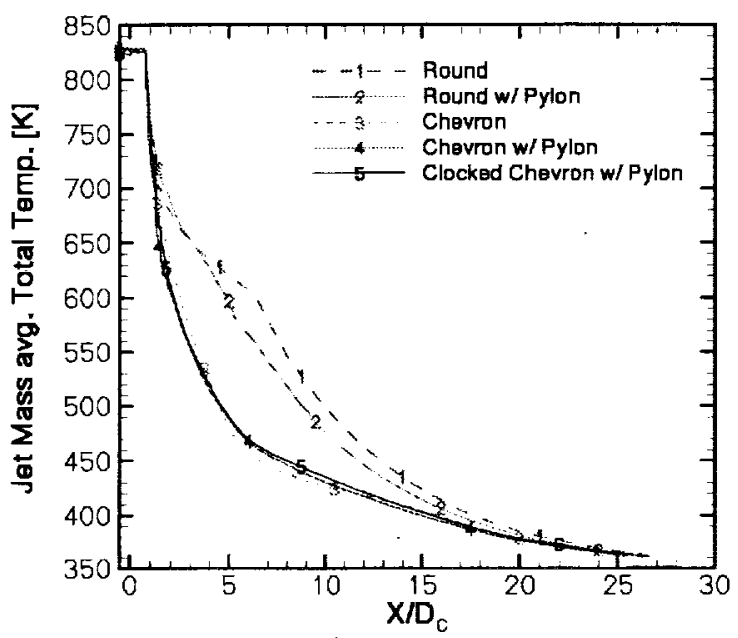

Figure 10. Mass averaged total temperature as a function of axial distance for configurations 1-5. Radial limit of integration at 0.5 -percent of fan temperature.

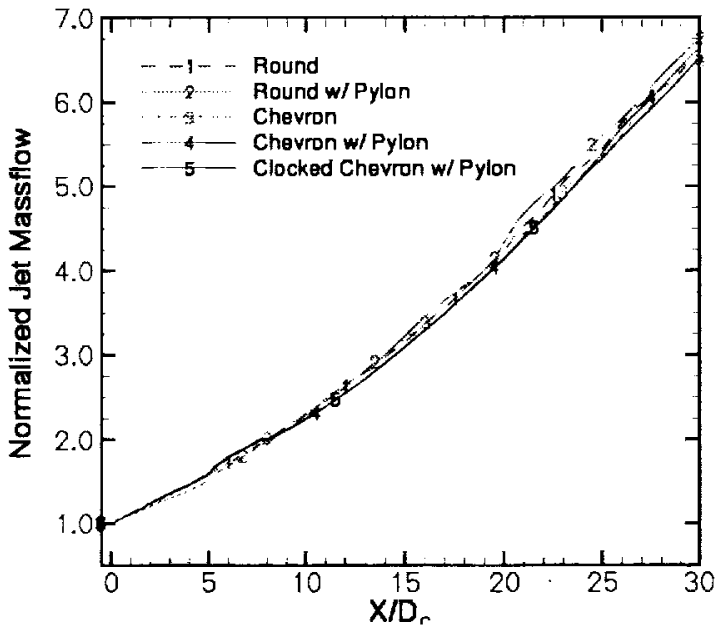

Figure 11. Normalized mass flow as a function of axial distance for configurations 1-5. Radial limit of integration at 0.5 -percent of ambient temperature.

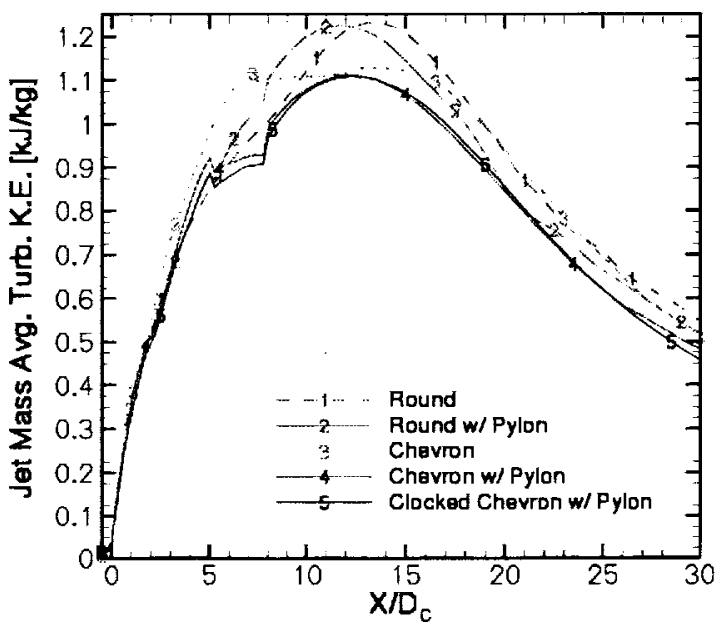

Figure 12. Mass averaged turbulent kinetic energy as a function of axial distance for configurations 1-5. Radial limit of integration at 0.5 -percent of ambient temnerature. 


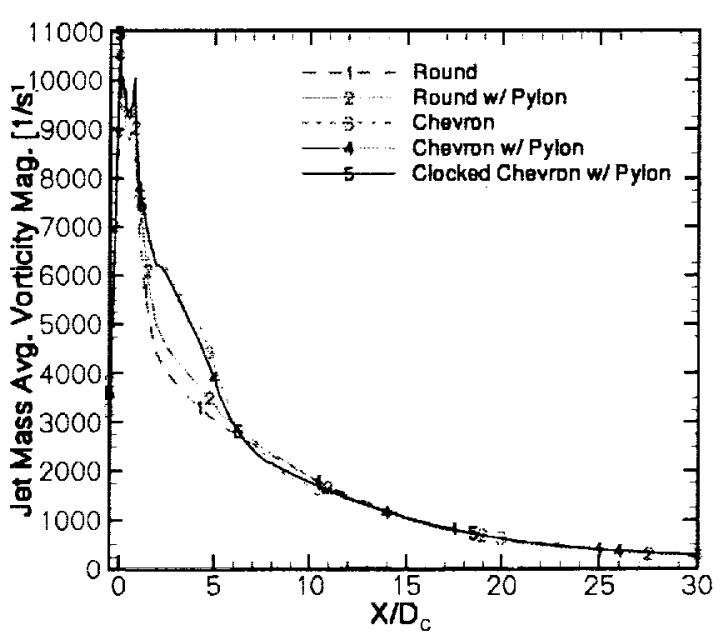

Figure 13. Mass averaged vorticity as a function of axial distance for configurations 1-5. Radial limit of integration at 0.5 -percent of ambient temperature.

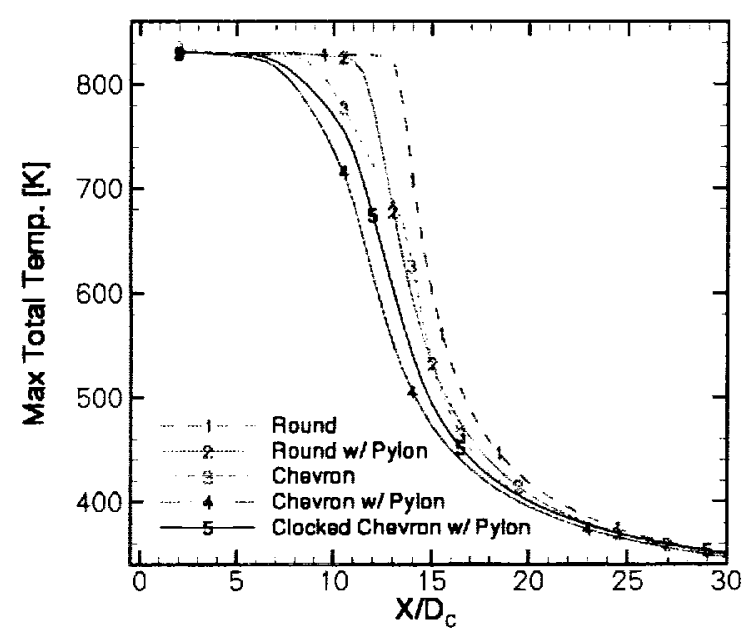

Figure 14. Maximum total temperature as a function of axial distance for configurations 1-5.

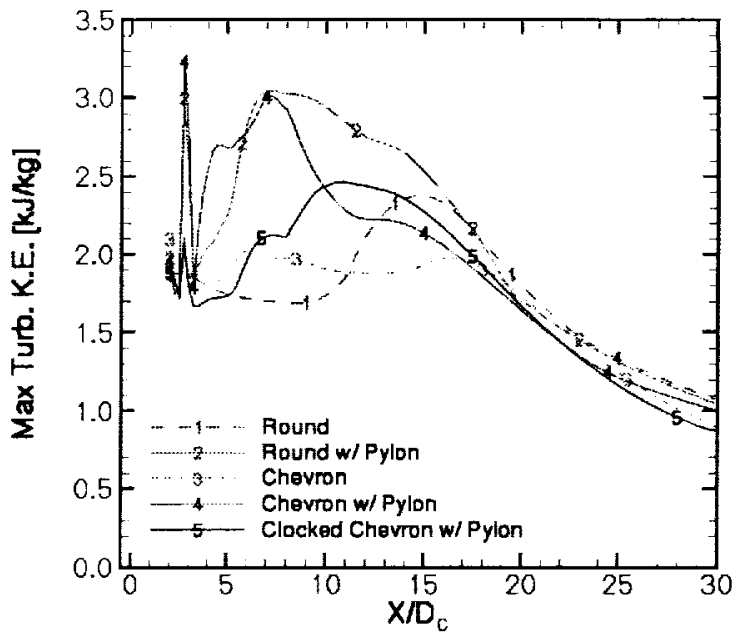

Figure 15. Maximum turbulent kinetic energy as a function of axial distance for configurations 1-5. 